\section{Incidencia de síndrome de Stevens-Johnson y necrólisis epidérmica tóxica en Chile años 2001-2015 y su asociación con latitud}

\author{
JAVIER ARELLANO ${ }^{1,2}$, DANAE ÁLVAREZ ${ }^{2,3}$, \\ MARÍA PAZ SALINAS ${ }^{\mathrm{a}}$, ILMA MOLINA ${ }^{\mathrm{a}}$
}

\section{Hospital discharges due to Stevens-Johnson syndrome and toxic epidermal necrolysis in Chile from 2001 to 2015}

Background: Stevens-Johnson Syndrome (SSJ) and Toxic Epidermal Necrolysis (NET) are infrequent and life-threatening mucocutaneous diseases, which occur predominantly as adverse drug reactions. Aim: To describe the frequency of SSJ and NET diagnoses at a national level, estimate their inciden$c e$ and describe their distribution among the different regions of the country. Material and Methods: Analysis of hospital discharge databases available at the website of the Chilean Ministry of Health searching for the tenth version of the International Classification of Diseases (ICD 10) codes for SSJ or NET, between 2001 and 2015. Results: We analyzed 24,521,796 hospital discharges nationwide. SSJ caused 855 discharges, with a lethality of $2 \%$. NET caused 128 discharges with a lethality of $16 \%$. The global cumulative incidence was 3.87 cases per million inhabitants per year nationwide, with a trend line to increase incidence towards the regions of higher latitude. Conclusions: SSJ and NET are dermatological emergencies with high mortality. The increase in incidence towards regions at higher latitudes may suggest an association between these conditions and lower levels of vitamin $D$, correlated with latitude and exposure to $U V$ radiation.

(Rev Med Chile 2020; 148: 915-920)

Key words: Drug Eruptions; Epidemiology; Stevens-Johnson Syndrome; Vitamin D.

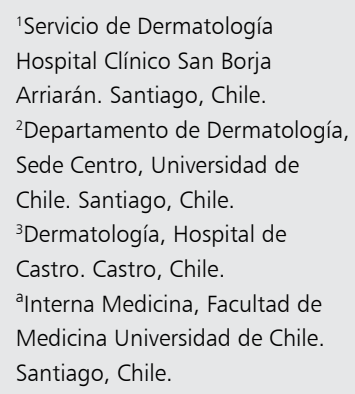

Los autores declaran no tener conflictos de interés.

Trabajo no recibió financiamiento.

Recibido el 18 de noviembre de 2019, aceptado el 3 de julio de 2020.

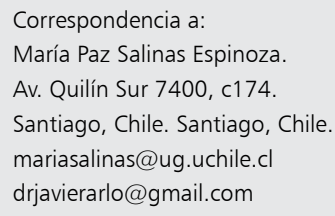

$\mathrm{E}$ 1 síndrome de Stevens-Johnson (SSJ) y la necrólisis epidérmica tóxica (NET) son enfermedades mucocutáneas infrecuentes y potencialmente mortales, que representan un espectro y ocurren predominantemente como reacciones adversas a medicamentos. Dependiendo del país de origen, la incidencia global de SSJ/ NET varía de 1,9 casos por millón en Europa, 6,5 casos por millón en Asia y, aproximadamente, 12 casos por millón en los Estados Unidos de Norteamérica9.
Tanto el SSJ como la NET se manifiestan con necrosis epidérmica y mucosa, sin embargo, difieren en la proporción de desprendimiento epidérmico. En el SSJ se presenta menos de 10\% de desprendimiento epidérmico, mientras que NET involucra más de 30\% del área de superficie corporal. La superposición SSJ/NET se utiliza para describir casos entre estos dos extremos ${ }^{8}$. Varios estudios observacionales han identificado los antiepilépticos, el alopurinol y ciertos antibióticos como los medicamentos con mayor riesgo de SSJ/ 
NET, aunque también se han asociado con menos evidencia analgésicos oxicam, sertralina, bloqueadores de la ciclooxigenasa, entre otros ${ }^{7}$. Ambas entidades son condiciones que amenazan la vida y que requieren atención médica inmediata. Para SSJ, la mortalidad estimada alcanza $10 \%$, menor que para NET, que se describe en 30 a $40 \%{ }^{10}$. En Estados Unidos de Noreteamérica (EE.UU.) se ha descrito una mortalidad de $4,8 \%$ para SSJ y $19,4 \%$ para NET ${ }^{11}$.

Hasta la fecha, en Chile no se han publicado estudios que analicen la incidencia de estas patologías a nivel nacional, existiendo solo reportes de centros hospitalarios individuales ${ }^{12,13}$. El análisis de egresos hospitalarios es un buen indicador de incidencia, ya que todos los pacientes con estos diagnósticos requieren hospitalización por la morbimortalidad asociada.

Chile es un país largo y angosto, con $4.329 \mathrm{~km}$ de longitud, y su área continental se extiende entre los $17^{\circ}$ y $56^{\circ}$ de latitud. Varios estudios han descrito la correlación que existe entre las concentraciones de vitamina D y diferentes latitudes (por consiguiente, con la intensidad de radiación), habiéndose demostrado que en ciudades del norte, como Arica (Latitud $18^{\circ} \mathrm{S}$ ), la proporción de hipovitaminosis D es menor comparado con Punta Arenas, ciudad del extremo sur del país (Latitud 520S) ${ }^{14-16}$. Múltiples estudios observacionales han demostrado que el déficit de vitamina $\mathrm{D}$ es un factor de riesgo en enfermedades inmunomediadas ${ }^{17,18}$.

El objetivo del presente trabajo es conocer la incidencia de pacientes con diagnósticos de SSJ y NET entre los años 2001 y 2015 a nivel nacional y su distribución sexo, edad, tiempo de estancia hospitalaria, condición de egreso y la distribución de la incidencia entre las distintas regiones del país.

\section{Método}

Se realizó un estudio ecológico transversal observacional con análisis del diagnóstico dermatológico al momento del egreso hospitalario entre los años 2001 y 2015. Los datos fueron obtenidos a partir de la base de datos del Departamento de Estadísticas e Información de Salud (DEIS), del Ministerio de Salud de Chile. Del total de pacientes hospitalizados a nivel nacional, se incluyeron aquellos registrados con el diagnóstico de SSJ o NET como primer diagnóstico de egreso, según la clasificación y codificación del CIE-10.
Para el análisis se consideraron las siguientes variables: diagnóstico, sexo, edad, tiempo de estancia hospitalaria, condición de egreso y región del país, basado en ubicación del centro hospitalario que notifica el caso.

La tasa de incidencia fue calculada considerando el número de egresos hospitalarios en el numerador y la población estimada para los años 2001 al 2015 como el denominador para cada región. Los datos de población estimada para cada año fueron obtenidos de la base de datos del Instituto Nacional de Estadísticas de Chile (INE) ${ }^{19}$.

El análisis estadístico se realizó con el programa IBM SPSS Statistics versión 20 (IBM, Armonk, NY). Se realizaron estimadores estadísticos descriptivos y de frecuencias para la descripción de las variables por separado. Se fijó una confianza de $95 \%$.

\section{Resultados}

Se analizaron 24.521.796 registros de egresos hospitalarios país entre los años 2001 y 2015, donde $2,45 \%$, equivalente a 600.012 registros, corresponde a patología dermatológica.

El síndrome de Stevens-Johnson presentó un total de 855 egresos y necrólisis epidérmica tóxica un total de 128 egresos, correspondiendo a $0,14 \%$ y $0,021 \%$ del total de egresos por patologías dermatológicas, respectivamente. La Tabla 1 resume los resultados de incidencia acumulada para SSJ y NET, según región del país.

De los pacientes con SSJ, 52,98\% correspondía a mujeres y $47,02 \%$ a hombres con una edad mínima de 0 y máxima de 100 años (promedio 36,12 \pm 23,465). El mínimo de estadía hospitalaria fue de 1 día y el máximo de 189 días, promedio 10 días \pm 10,81. El 97,7\% de los egresos fueron en condición vivo y $2,3 \%$ fallecido ( 20 fallecidos de 855 egresos totales). A partir de los egresos hospitalarios, se estima que la incidencia acumulada de SSJ a nivel nacional para el período 2001-2015 correspondía a 3,36 casos nuevos por millón de habitantes al año. La mayor incidencia se presentó en la Región de Magallanes, con 4,61 casos por millón de habitantes al año, mientras que la menor incidencia se observa en la Región de Atacama, con una incidencia de 2,13 casos por millón de habitantes al año. La Figura 1 muestra la distribución de la incidencia acumulada según región del país, con la línea de tendencia según latitud. 
Incidencia de SSJ y NET en Chile y asociación con latitud - J. Arellano et al

Tabla 1. Incidencia acumulada de síndrome de Steven-Johnson y necrolisis epidérmica tóxica en Chile, según región

\begin{tabular}{|ccccccc|}
\hline Región & $\begin{array}{c}\text { Población total } \\
\text { período 2001-2015 }\end{array}$ & $\begin{array}{c}\text { Casos SSJ } \\
(\mathbf{n})\end{array}$ & $\begin{array}{c}\text { Casos NET } \\
(\mathbf{n})\end{array}$ & $\begin{array}{c}\text { Incidencia } \\
\text { acumuada SSJ* }\end{array}$ & $\begin{array}{c}\text { Incidencia } \\
\text { acumulada NET* }\end{array}$ & $\begin{array}{c}\text { Incidencia } \\
\text { SSJ/NET* }\end{array}$ \\
\hline 1 & 7.407 .920 & 21 & 3 & 2,834 & 0,404 & 3,239 \\
\hline 2 & 8.227 .983 & 18 & 3 & 2,187 & 0,364 & 2,552 \\
\hline 3 & 4.207 .401 & 9 & 1 & 2,139 & 0,237 & 2,376 \\
\hline 4 & 10.338 .032 & 24 & 4 & 2,321 & 0,386 & 2,708 \\
\hline 5 & 25.663 .847 & 94 & 9 & 3,662 & 0,350 & 4,013 \\
\hline RM & 101.385 .952 & 383 & 52 & 3,777 & 0,512 & 4,290 \\
\hline 6 & 12.964 .973 & 47 & 15 & 3,625 & 1,156 & 4,782 \\
\hline 7 & 14.967 .568 & 43 & 2 & 2,872 & 0,133 & 3,006 \\
\hline 8 & 30.257 .394 & 86 & 14 & 2,842 & 0,462 & 3,304 \\
\hline 9 & 14.082 .956 & 39 & 17 & 2,769 & 1,207 & 3,976 \\
\hline 10 & 17.658 .474 & 66 & 7 & 3,737 & 0,396 & 4,133 \\
\hline 11 & 1.475 .285 & 4 & 1 & 2,711 & 0,677 & 3,389 \\
\hline 12 & 2.385 .390 & 11 & 0 & 4,611 & 0 & 4,611 \\
\hline Total & 251.023 .175 & 845 & 128 & 3,366 & 0,509 & 3,876 \\
\hline
\end{tabular}

*Incidencia acumulada: número de casos por millón de habitantes. RM: Región Metropolitana. SSJ: síndrome de StevensJohnson. NET: necrólisis epidérmica tóxica.

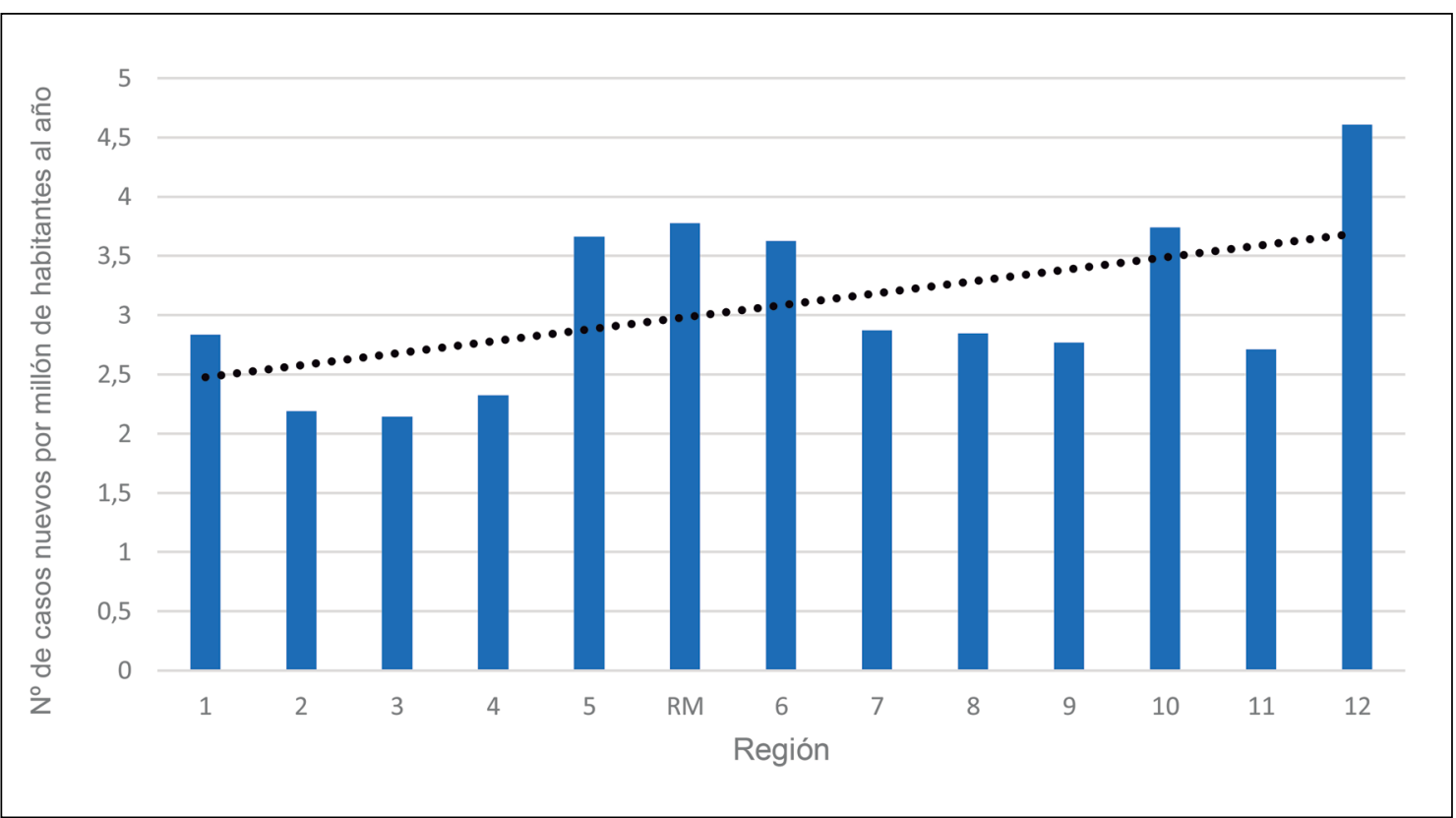

Figura 1. Incidencia acumulada de síndrome de Stevens-Johnson por millón de habitantes al año para el período 2001-2015, según región del país. 


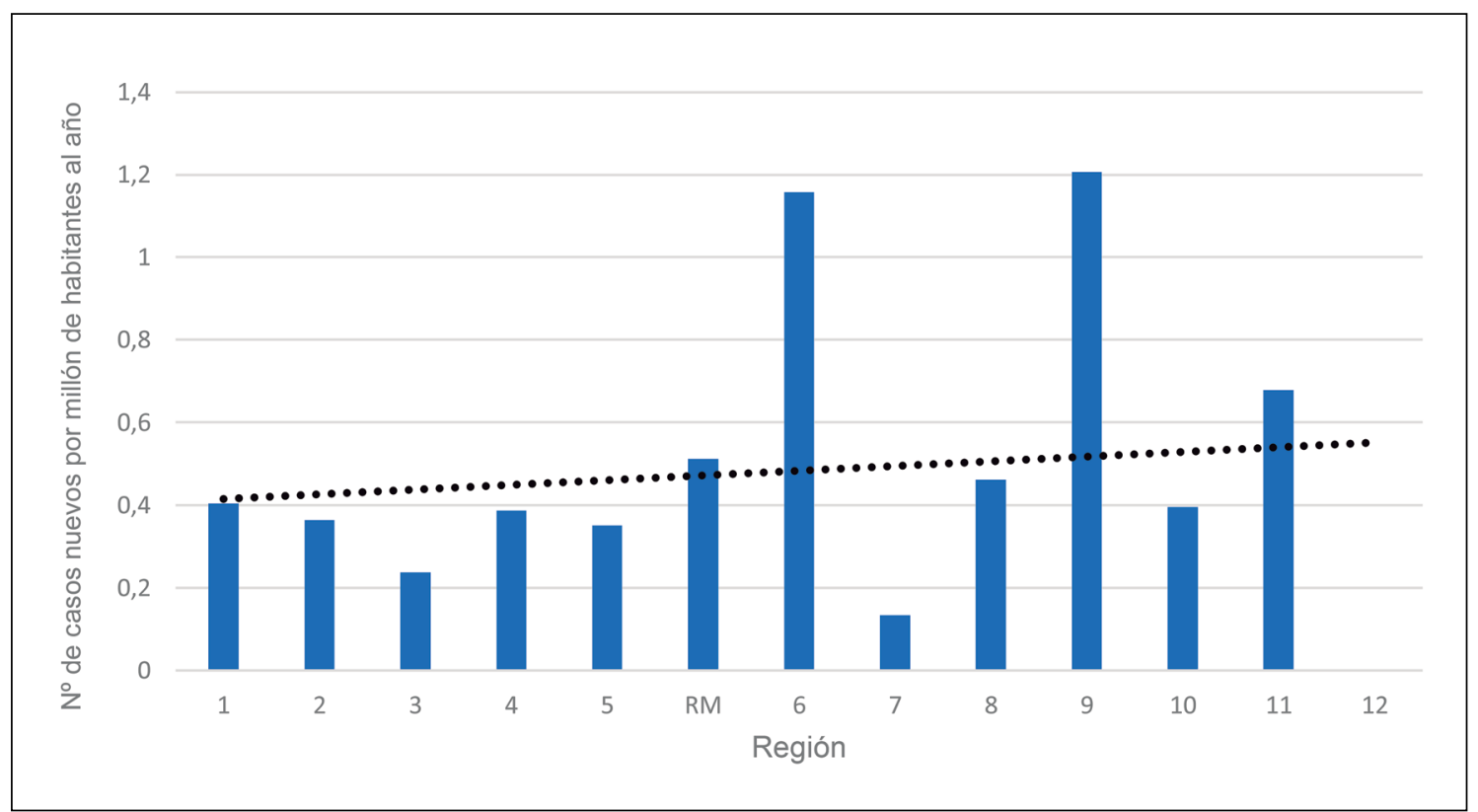

Figura 2. Incidencia acumulada de necrólisis epidérmica tóxica por millón de habitantes al año para el período 2001-2015, según región del país.

Del subgrupo NET, 58,59\% correspondía a mujeres y $41,41 \%$ a hombres. La edad mínima fue de 0 y máxima de 92 años (promedio 37,87 \pm $24,825)$, con 1 día mínimo de estadía y un máximo de 96 días, promedio 18,55 días \pm 17,45. El 84,4\% de los egresos se registraron en condición vivo y $15,6 \%$ fallecido (20 fallecidos de 128 egresos totales). La estimación de incidencia acumulada de NET a nivel país es de 0,51 casos por millón de habitantes para el período 2001-2015. La mayor incidencia se observó en la Región de Los Lagos, con 1,21 casos por millón de habitantes al año, mientras que la menor incidencia se obtuvo para la Región de Magallanes con 0 casos por millón de habitantes durante el perídodo 2001-2015. La Figura 2 muestra la incidencia acumulada de NET por millón de habitantes al año, para el período 2001-2015, según regiones del país y con la línea de tendencia según latitud.

\section{Discusión}

El presente estudio analiza la frecuencia, distribución e incidencia acumulada SSJ y NET durante el período 2001-2015, a través de los informes de egresos hospitalarios. Al ser patologías cuyo tratamiento siempre se realiza a nivel hospitalario, el número de egresos refleja el total de pacientes que han desarrollado la enfermedad en un determinado período, por lo que constituye un buen estimador de incidencia. Debido a que en Chile es obligatorio mantener registro de los diagnósticos de egreso de todos los pacientes hospitalizados y su reporte al DEIS-MINSAL, esta metodología resulta adecuada para obtener una estimación de incidencia a nivel nacional y regional. $\mathrm{Al}$ analizar los resultados, se observa una incidencia de ambas patologías de 3,87 casos por millón de habitantes al año a nivel nacional, con una mayor incidencia de SSJ en comparación con NET, cifras similares a las reportadas en la literatura internacional ${ }^{9}$. Destaca el aumento progresivo de la incidencia en relación con la latitud, observándose una tendencia creciente desde las regiones del norte hacia regiones del sur de Chile. $\mathrm{Si}$ bien en este estudio no se analizan factores causales de este fenómeno, los resultados aquí expuestos permiten plantear que un aumento en la latitud, asociado a menor exposición a radiación 
UV y, por consiguiente, menores niveles de vitamina $\mathrm{D}$, como ha sido demostrado en estudios previos $^{14-16}$, podría determinar un aumento en el desarrollo de estas patologías, basado en los mecanismos inmunomediados involucrados en su patogenia. Es necesario realizar nuevos estudios de base individual que permitan confirmar esta asociación y una eventual relación causal entre estos factores. El SSJ y NET son patologías que presentan una importancia clínica particular, ya que corresponden a una urgencia dermatológica con alta mortalidad, entre 1 y $5 \%$ para SSJ y 25 y $35 \%$ para $\mathrm{NET}^{20}$. Además, los sobrevivientes, a menudo, también experimentan una gran cantidad de secuelas a largo plazo, incluyendo cambios en la pigmentación y cicatrización de la piel, síntomas sicca, discapacidad visual, enfermedad periodontal, ulceración gastrointestinal, y estenosis vaginal y uretral, así como aumento de autoinmunidad ${ }^{11}$.

Respecto a la mortalidad, en el presente estudio se observó una mayor proporción de pacientes egresados en condición de fallecidos con diagnóstico de NET en comparación con SSJ. Esto concuerda con lo descrito en la literatura respecto a los valores de mortalidad para SSJ, y la mayor mortalidad que presenta NET, lo que tiene relación al compromiso más extenso de la superficie corporal y mayor gravedad de esta última. Sin embargo, destaca una mortalidad menor a la descrita en la literatura para NET, $15,6 \%$ en este estudio, en comparación con 25 a $35 \%$ previamente descritos ${ }^{20}$.

Con este trabajo se busca avanzar en la caracterización epidemiológica del SSJ y NET, dando a conocer los datos existentes a nivel nacional y regional. Dentro de las limitaciones, existen los sesgos inherentes al sistema de codificación CIE-10, que al ser múltiples digitadores incluye errores de digitalizacion, falta de identificación o prioridad de diagnósticos, entre otros. Pese a lo anterior, los resultados de este estudio constituyen un aporte significativo dada la escasa literatura disponible al respecto, tanto nacional como internacional, pudiendo contribuir a la creación y optimización de políticas públicas para mejorar la atención de los pacientes con patologías dermatológicas y realizar evantualmente nuevos estudios que permitan identificar a la vitamina $\mathrm{D}$ como una factor importante en el desarrollo de esta condicion inmunomediada.

\section{Referencias}

1. Hay RJ, Fuller LC. The assessment of dermatological needs in resource- poor regions. Int J Dermatol 2011; 50 (5): 552-7.

2. Fernandes IC, Velho G, Selores M. Dermatology inpatient consultation in a Portuguese university hospital. Dermatol Online J 2012; 18 (6): 16.

3. Kirsner RS, Yang DG, Kerdel FA. Dermatologic disease accounts for a large number of hospital admissions annually. J Am Acad Dermatol 1999; 41 (6): 970-3.

4. Biesbroeck LK, Shinohara MM. Inpatient Consultative Dermatology. Med Clin North Am 2015; 99 (6): 1349-64.

5. Davila M, Christenson LJ, Sontheimer RD. Epidemiology and outcomes of dermatology in-patient consultations in a Midwestern U.S. university hospital. Dermatol Online J 2010; 16 (2): 12.

6. Connolly DM, Silverstein DI. Dermatology consultations in a tertiary care hospital: A retrospective study of 243 cases. Dermatol Online J 2015; 21 (8).

7. Wong A, Malvestiti AA, Hafner Mde F. Stevens-Johnson syndrome and toxic epidermal necrolysis: a review. Rev Assoc Med Bras 2016; 62 (5): 468-73.

8. Woolum J, Bailey A, Barum R, Metts E. A Revew of the Management of Stevens-Johnson Syndrome and Toxic Epidermal Necrolysis. Advanced Emergency Nursing Journal 2019; 41 (1): 56-64.

9. Mustafa S, Ostrov D, Yerly D. Severe Cutaneous Adverse Drug Reactions: Presentation, Risk Factors and Management. Curr Allergy Asthma Rep 2018; 18: 26.

10. Sekula P, Dunant A, Mockenhaupk M, Naldi L, Bouwes-Bavinck JN, Halevy S, et al. Comprehensive survival analysis of a cohort of patients with Stevens-Johnson syndrome and toxic epidermal necrolysis. J Invest Dermatol 2013; 133 (5): 1197-204.

11. Hsu DY, Brieva J, Silverberg NB, Silverberg JI. Morbidity and mortality of Stevens-Johnson syndrome and toxic epidermal necrolysis in United States adults. J Invest Dermatol 2016; 136 (7): 1387-97.

12. Fuenzalida H, Cardemil S, Cardemil A. Morbilidad dermatológica en interconsultas de pacientes hospitalizados en el Complejo Asistencial Barros Luco. Rev. Chilena Dermatol 2011; 27 (2): 162-9.

13. Villagrán B, Calderón P, Espinoza M. Patología cutánea en pacientes hospitalizados del Hospital Clínico de la Universidad de Chile. Rev Chil Dermatol 2007; 23 (3): 188-91.

14. Holick, MF. Vitamin D deficiency. N Engl J Med 2007; 357: 266-81.

15. Rodríguez J. Valdivia G, Trincado P. Fracturas vertebrales, osteoporosis y vitamina $\mathrm{D}$ en la posmenopausia: 
Estudio en 555 mujeres en Chile. Rev Med Chile 2007; 135: 31-6.

16. Brinkmann K, LeRoy C, Íñiguez G, Borzutzky A. Deficiencia severa de vitamina D en niños de Punta Arenas, Chile: influencia de estado nutricional en la respuesta a suplementación. Rev Chil Ped 2015; 86: 182-8.

17. García-Carrasco M, Jiménez-Herrera EA, Gálvez-Romero JL, de Lara LV, Mendoza-Pinto C, Etchegaray-Morales I, et al. Vitamin D and Sjögren syndrome. Autoimmun Rev 2017; 16: 587-93.

18. Mautorri N, Cantatore F. Vitamin D and the immune system. J Rheumatol 2007; 37: 491-5.

19. Instituto Nacional de Estadísticas INE. Estimaciones y proyecciones de la población de Chile 2002-2035 regiones y área urbano rural. Disponibe en: https://www.ine. cl/estadisticas/demograficas-y-vitales (consultado el 30 de agosto 2019).

20. Miliszewski MA, Kirchhof MG, Sikora S, Papp A, Dutz JP. Stevens-Johnson Syndrome and Toxic Epidermal Necrolysis: An Analysis of Triggers and Implications for Improving Prevention. Am J Med 2016; 129 (11): 1221-5. 